\title{
Bifurcation Analysis of a Discrete-Time Four-Dimensional Cubic Autocatalator Chemical Reaction Model with Coupling Through Uncatalysed Reactant
}

\author{
Muhammad Salman Khan \\ Department of Mathematics, Quaid-I-Azam \\ University Islamabad, 44230, Pakistan, \\ mskhan@math.qau.edu.pk
}

(Received April 10, 2021)

\begin{abstract}
In this manuscript, we discuss a four-dimensional cubic autocatalator chemical reaction model in continuous form. We investigate the existence of one and only positive fixed point and then we have obtained some parametric conditions for local stability of continuous system by using Routh-Hurwitz stability criteria. Moreover, we discretize the four-dimensional continuous cubic autocatalator chemical reaction model by using Euler's forward method and then by using a nonstandard difference scheme we obtained a consistent discrete-time counterpart of four-dimensional cubic autocatalator chemical reaction model. Parametric conditions for local asymptotic stability of one and only positive fixed point of obtained system are also discussed. It is shown that the obtained system experiences the Neimark-Sacker bifurcation at one and only positive fixed point by using a general standard for Neimark-Sacker bifurcation. The discrete-time counterpart of genuine four-dimensional system displays chaotic dynamics at different standards of bifurcation parameter. Furthermore, the control of Neimark-Sacker bifurcation and chaos is also deliberated by using a generalized hybrid control scheme, which is based on parameter perturbation and feedback control. Finally, some numerical examples are given to strengthen our theoretical results.
\end{abstract}




\section{Introduction}

In this article we study a prototype two-cell model using the feedback scheme in every cell being centered on the cubic autocatalator reaction, which is listed as

$$
\begin{cases}A+2 B \rightarrow 3 B & \text { rate } k_{0} p, \\ P \rightarrow A & \text { rate } k_{1} a b^{2}, \\ B \rightarrow C & \text { rate } k_{2} b,\end{cases}
$$

with the following relation for uncatalysed phase

$$
\left\{A \rightarrow B \quad \text { rate } k_{3} a,\right.
$$

(where, the concentrations of the reactants $A, B$ and $P$ are respectively represented by $a, b$ and $p[1])$. In addition, $k_{i}(i=0,1,2,3)$ are rate constants. We start with the condition that there is a semi-permeable coating between the cells which permits diffusion of only one from the reactant classes $B$ or $A$ at any instant. Moreover, the overall reaction is feeded by using the condition that reactant $A$ is produced by only first-order decline from its originator $P$. It is assumed that the production of $A$ from its originator $P$ at the rate $k_{1} a b^{2}$ is comparatively slow process with the following supposition that the primary concentration of $P$, explicitly $p_{0}$, is numerous orders of size larger than that of the intermediates $B$ and $A[1]$. In these conditions it is standard to consider the 'pooledchemical' estimations and make the additional supposition that the concentration of $P$ remains same all the way through, at its primary value $p_{0}$. Additionally, it is considered that the rate-defining stage, namely, basic autocatalytic step $(A+2 B \rightarrow 3 B)$ is faster when compared to uncatalysed reaction step. Yet, this additional reaction can have a significant influence on the total behavior of the system. Few decades earlier the authors in [3-8] have widely studied the separated reaction model (1) in a well-stirred scheme under the effects of pooled-chemical estimation. The authors in [8] and [2] have revealed that this reaction system may experience some complex designs of behavior. The authors in [11] have discussed the consequences of slow decline of the antecedent $P$ on the overall solution. The authors in [1] have extended the work of [12] on this dual-cell problem centered on the cubic-autocatalator response system (1). Moreover, in their extended work they have discussed some consequences that the additional uncatalysed response stage (2) had on the inclusive behavior of the reaction. Merkin et al. [6] have showed that with a comparatively small influence, this additional step can ensure a key effect on 
the result of the uncoupled system by keeping the result restricted, by not letting the concentration of $B$ to approach to zero (although it can turn out to be negligible). The addition of this additional stage to the uncoupled system have extreme impact on the oscillatory behavior, keeping the oscillatory response restricted all over and giving rise to an additional point of Hopf bifurcation.

A complete study of complexity in the dynamical behavior, wherever the noncatalysed stage was not involved, is presented in the article [12]. Moreover, the authors in [1] have not presented the complete study of complexity in the dynamical behavior that can ascend due to secondary bifurcations. The equations leading this dual-cell combined system are, under the pooled-chemical estimate for the reactant $P$ (see [1]),

$$
\begin{aligned}
& \frac{d a_{1}}{d t_{1}}=k_{0} p_{0}-k_{1} b_{1}^{2} a_{1}-k_{3} a_{1}+D_{a}\left(a_{2}-a_{1}\right), \\
& \frac{d b_{1}}{d t_{1}}=k_{1} b_{1}^{2} a_{1}+k_{3} a_{1}-k_{2} b_{1}+D_{b}\left(b_{2}-b_{1}\right), \\
& \frac{d a_{2}}{d t_{1}}=k_{0} p_{0}-k_{1} b_{2}^{2} a_{2}-k_{3} a_{2}+D_{a}\left(a_{1}-a_{2}\right), \\
& \frac{d b_{2}}{d t_{1}}=k_{1} b_{2}^{2} a_{2}+k_{3} a_{2}-k_{2} b_{2}+D_{b}\left(b_{1}-b_{2}\right) .
\end{aligned}
$$

Where, the diffusion coefficients for autocatalyst $B$ and reactant $A$ are $D_{b}$ and $D_{a}$ respectively through the membrane separating the two cells. Furthermore, to made the nondimensional system of equations from (3), we consider the following transformations [12]:

$$
\left\{\begin{array}{l}
x_{i}=a_{i} \sqrt{\frac{k_{1}}{k_{2}}}, \\
y_{i}=b_{i} \sqrt{\frac{k_{1}}{k_{2}}}, \\
(i=1,2), t=k_{2} t_{1} .
\end{array}\right.
$$

The terms arising from the decline of the antecedent $P$ give growth to a positive dimensionless parameter

$$
\mu=\frac{k_{0} p_{0}}{k_{2}} \sqrt{\frac{k_{1}}{k_{2}}} .
$$

The addition of uncatalysed stage give rise to a dimensionless parameter $r=\frac{k_{3}}{k_{2}}$, which will, in common, take comparatively lesser values. The combination through $B$ and $A$ give rise to the real numbers $\beta=\frac{D_{b}}{k_{2}}$ and $\alpha=\frac{D_{a}}{k_{2}}$ respectively. Moreover, under these 
assumptions the system (3) takes the following form;

$$
\begin{aligned}
& \frac{d x_{1}}{d t}=\mu-x_{1} y_{1}^{2}-r x_{1}+\alpha\left(x_{2}-x_{1}\right), \\
& \frac{d y_{1}}{d t}=x_{1} y_{1}^{2}-y_{1}+r x_{1}+\beta\left(y_{2}-y_{1}\right), \\
& \frac{d x_{2}}{d t}=\mu-x_{2} y_{2}^{2}-r x_{2}+\alpha\left(x_{1}-x_{2}\right), \\
& \frac{d y_{2}}{d t}=x_{2} y_{2}^{2}-y_{2}+r x_{2}+\beta\left(y_{1}-y_{2}\right),
\end{aligned}
$$

with $x_{i}, y_{i} \geq 0$ for every value of $i$ (see [12]). Some aspects of the common case $\alpha \neq \beta \neq 0$ for system (5) are considered by Askhenaz et al. [13]. Moreover, the authors in [1] have discussed some qualitative results of system (5) by letting pairing either through $B$ or through $A$ only. Formerly, it was explained by Lech et al. [1] that, when pairing is considered by using reactant $A$ only, we must have to take $\beta=0$, similarly for pairing through autocatalyst $B$ we have to take $\alpha=0$ in system (5). Here, we consider the coupling in system (5) through autocatalyst $B$ by taking $\alpha=0$. In this case the system (5) takes the following form:

$$
\begin{aligned}
& \frac{d u}{d t}=\mu-u v^{2}-r u, \\
& \frac{d v}{d t}=u v^{2}+r u-v+\beta(z-v), \\
& \frac{d w}{d t}=\mu-w z^{2}-r w, \\
& \frac{d z}{d t}=w z^{2}+r w-z+\beta(v-z),
\end{aligned}
$$

where $x_{1}=u, x_{2}=w, y_{1}=v$ and $y_{2}=z$. Recently, Din [14] have discussed the rich dynamics of discrete-time version of a glycolysis model. Moreover, Din et al. [15] have considered a discrete-time chlorine dioxide-iodine-malonic acid model for study and discussed the complexity in that mathematical system. In case of non-intersecting generations, discrete-time mathematical systems gives efficient computational results as compared to their counterparts in continuous form [16]. Mostly, global character, boundedness, local asymptotic stability, persistence, and the presence of positive periodic results can be argued more effortlessly in case of discrete-time systems as paralleled to their counterparts in continuous form [17]. There are various mathematical techniques for converting the differential equations to corresponding discrete counterparts. To achieve this goal the usual way is to apply standard difference schemes such as Runge-Kutta methods and Euler approximations. But, numerical inconsistency is experienced with the application 
of usual finite difference methods. Hence, to avoid this numerical inconsistency, one can apply nonstandard finite difference method given by Mickens [18]. In general, whenever a nonstandard finite difference scheme is proposed then it is aimed on the preservation of the following properties of the respective continuous-time system: positivity of results, boundedness, stability of equilibrium points, existence and nature of bifurcations. Additionally, the main benefit of the nonstandard finite difference schemes is to maintain the significant characters of their respective continuous systems. Moreover, the formation of these type of difference schemes is not straightforward and there are no usual way for their construction, which is probably considered as major downside of nonstandard difference schemes [29]. Hence, it is interesting to study the dynamics of discrete-time version of (6). Formally, by using Euler's forward method we get the following discrete-time version of system (6);

$$
\begin{aligned}
& u_{n+1}=u_{n}+\eta\left(a-u_{n} v_{n}^{2}-b u_{n}\right), \\
& v_{n+1}=v_{n}+\eta\left(u_{n} v_{n}^{2}+b u_{n}-v_{n}+c\left(z_{n}-v_{n}\right)\right), \\
& w_{n+1}=w_{n}+\eta\left(a-w_{n} z_{n}^{2}-b w_{n}\right), \\
& z_{n+1}=z_{n}+\eta\left(w_{n} z_{n}^{2}+b w_{n}-z_{n}+c\left(v_{n}-z_{n}\right)\right),
\end{aligned}
$$

where $0<\eta<1$. To understand similar type of discretization, one can study [15-18]. Moreover, by applying nonstandard finite difference scheme ( see Mickens [18]) we get the following form of system (7).

$$
\begin{aligned}
u_{n+1} & =\frac{\left(u_{n}+\eta \mu\right)}{\left(1+\eta\left(v_{n}^{2}+r\right)\right)}, \\
v_{n+1} & =\frac{v_{n}+\eta\left(u_{n} v_{n}^{2}+r u_{n}+\beta z_{n}\right)}{(1+\eta(1+\beta))}, \\
w_{n+1} & =\frac{\left(w_{n}+\eta \mu\right)}{\left(1+\eta\left(z_{n}^{2}+r\right)\right)}, \\
z_{n+1} & =\frac{\left(z_{n}+\eta\left(w_{n} z_{n}^{2}+r w_{n}+\beta v_{n}\right)\right)}{(1+\eta(1+\beta))} .
\end{aligned}
$$

Rest of this paper is aimed at the study of the existence of one and only fixed point of system (8). The local stability of system (8) about one and only positive fixed point of system (8). The existence of Neimark-Sacker bifurcation about one and only positive fixed point of system (8). In concern to control the chaos under the effects of bifurcation, we apply a modified hybrid control approach to system (8). In last section, some numerical examples are also provided. 


\section{Existence of fixed points and linearized stability of system (8)}

From system (8) one can get the one and only fixed point $\left(u_{*}, v_{*}, w_{*}, z_{*}\right)$. Additionally, $\left(u_{*}, v_{*}, w_{*}, z_{*}\right)$ represents the unique positive fixed point of system (8). Furthermore, this equilibrium point is given as:

$$
\left(u_{*}, v_{*}, w_{*}, z_{*}\right)=\left(\frac{\mu}{r+\mu^{2}}, \mu, \frac{\mu}{r+\mu^{2}}, \mu\right) .
$$

In order to study the stability analysis of system (8)about one and only positive fixed point, we have the next theorem, which provides us freely applicable necessary and sufficient conditions for all the roots of real fourth order polynomial to have magnitude less than one (see, Theorem 1.5 of [19]).

Theorem 2.1. Assume the fourth degree characteristic equation

$$
\rho^{4}+d_{1} \rho^{3}+d_{2} \rho^{2}+d_{3} \rho+d_{4}=0
$$

where $d_{1}, d_{2}, d_{3}, d_{4} \in \Re$. Additionally, let $\rho_{1}, \rho_{2}, \rho_{3}, \rho_{4}$ are roots of (9) and $D_{1}$ be any open disk of radius one. Then, the necessary and sufficient conditions that $\rho_{1}, \rho_{2}, \rho_{3}, \rho_{4} \in D_{1}$ are given as:

$$
\left|d_{3}+d_{1}\right|<1+d_{4}+d_{2}, \quad\left|d_{3}-d_{1}\right|<2\left(1-d_{4}\right), \quad d_{2}-3 d_{4}<3,
$$

and

$$
d_{4}+d_{2}+d_{4}^{2}+d_{3}^{2}+d_{4}^{2} d_{2}+d_{4} d_{1}^{2}<1+2 d_{4} d_{2}+d_{3} d_{1}+d_{4} d_{3} d_{1}+d_{4}^{3}
$$

Theorem 2.2. Consider the following biquadratic polynomial equation with real coefficients

$$
\rho^{4}+d_{1} \rho^{3}+d_{2} \rho^{2}+d_{3} \rho+d_{4}=0
$$

Then, necessary and sufficient conditions that all the roots of (10) lie inside the disk of unit radius are given as follows:

$$
\left\{\begin{array}{l}
\left|d_{3}+d_{1}\right|<1+d_{4}+d_{2}, \\
\left|d_{3}-d_{1}\right|<2\left(1-d_{4}\right) \\
d_{2}-3 d_{4}<3 \\
d_{4}+d_{2}+d_{4}^{2}+d_{3}^{2}+d_{4}^{2} d_{2}+d_{4} d_{1}^{2}<1+2 d_{4} d_{2}+d_{3} d_{1}+d_{4} d_{3} d_{1}+d_{4}^{3} .
\end{array}\right.
$$


where

$$
\left\{\begin{array}{l}
d_{1}=-\left(\frac{2}{1+S \eta}+\frac{2\left(S+2 \eta \mu^{2}\right)}{S T}\right), \\
d_{2}=\frac{\frac{\left(S+2 \eta \mu^{2}\right)^{2}}{S^{2}}+\frac{S T(4+T+4 S \eta)+4 T \eta(1+S \eta)(2+S \eta) \mu^{2}}{S(1+S \eta)^{2}}-\beta^{2} \eta^{2}}{T^{2}}, \\
d_{3}=\frac{2 S^{2}\left((1+S \eta)\left(1-\beta^{2} \eta^{2}\right)-T\right)-4 S \eta(1+S \eta)(2+T+S \eta) \mu^{2}-8 \eta^{2}(1+S \eta)^{2} \mu^{4}}{S^{2} T^{2}(1+S \eta)^{2}} \\
d_{4}=\frac{S^{2}\left(1-\beta^{2} \eta^{2}\right)+4 S \eta(1+S \eta) \mu^{2}+4 \eta^{2}(1+S \eta)^{2} \mu^{4}}{S^{2} T^{2}(1+S \eta)^{2}} \\
S=r+\mu^{2} \text { and } T=1+\eta+\beta \eta .
\end{array}\right.
$$

Proof. Let $J_{\left(u_{*}, v_{*}, w_{*}, z_{*}\right)}$ be the jacobian matrix of system (8) about $\left(\frac{\mu}{r+\mu^{2}}, \mu, \frac{\mu}{r+\mu^{2}}, \mu\right)$ then $J_{\left(u_{*}, v_{*}, w_{*}, z_{*}\right)}$ has the following mathematical form

$$
J_{\left(u_{*}, v_{*}, w_{*}, z_{*}\right)}=\left(\begin{array}{cccc}
\frac{1}{1+\eta S} & -\frac{2 \eta \mu^{2}}{S(1+\eta S)} & 0 & 0 \\
\frac{\eta S}{T} & \frac{1+\frac{2 \eta \mu^{2}}{S}}{T} & 0 & \frac{\beta \eta}{T} \\
0 & 0 & \frac{1}{1+\eta S} & -\frac{2 \eta \mu^{2}}{S\left(1+\eta S^{2}\right)} \\
0 & \frac{\beta \eta}{T} & \frac{\eta S}{T} & \frac{1+\frac{2 \eta \mu^{2}}{S}}{T}
\end{array}\right) .
$$

Moreover, the characteristic equation of $J_{\left(u_{*}, v_{*}, w_{*}, z_{*}\right)}$ is given as

$$
\rho^{4}+d_{1} \rho^{3}+d_{2} \rho^{2}+d_{3} \rho+d_{4}=0
$$

where $d_{1}, d_{2}, d_{3}, d_{4}$ are given in (12). Finally, by applying Theorem 2.2 , the one and only positive fixed point $\left(\frac{\mu}{r+\mu^{2}}, \mu, \frac{\mu}{r+\mu^{2}}, \mu\right)$ remains stable locally asymptotically if the following conditions are fulfilled:

$$
\left|d_{3}+d_{1}\right|<1+d_{4}+d_{2}, \quad\left|d_{3}-d_{1}\right|<2\left(1-d_{4}\right), \quad d_{2}-3 d_{4}<3,
$$

and

$$
d_{4}+d_{2}+d_{4}^{2}+d_{3}^{2}+d_{4}^{2} d_{2}+d_{4} d_{1}^{2}<1+2 d_{4} d_{2}+d_{3} d_{1}+d_{4} d_{3} d_{1}+d_{4}^{3}
$$

\section{Stability analysis of system (6)}

Assume that $J^{*}\left(u_{*}, v_{*}, w_{*}, z_{*}\right)$ be jacobian matrix of system (6) about $\left(\frac{\mu}{r+\mu^{2}}, \mu, \frac{\mu}{r+\mu^{2}}, \mu\right)$ then $J^{*}\left(u_{*}, v_{*}, w_{*}, z_{*}\right)$ has the following mathematical form:

$$
J^{*}\left(u_{*}, v_{*}, w_{*}, z_{*}\right)=\left(\begin{array}{cccc}
-r-\mu^{2} & -\frac{2 \mu^{2}}{r+\mu^{2}} & 0 & 0 \\
r+\mu^{2} & 1-\beta-\frac{2 r}{r+\mu^{2}} & 0 & \beta \\
0 & 0 & -r-\mu^{2} & -\frac{2 \mu^{2}}{r+\mu^{2}} \\
0 & \beta & r+\mu^{2} & 1-\beta-\frac{2 r}{r+\mu^{2}}
\end{array}\right) .
$$


Additionally, let $F(\xi)=0$ be characteristic equation obtained from $J^{*}\left(u_{*}, v_{*}, w_{*}, z_{*}\right)$ then $F(\xi)=0$ can be specified as follows

$$
c_{4} \xi^{4}+c_{3} \xi^{3}+c_{2} \xi^{2}+c_{1} \xi+c_{0}=0
$$

where

$$
\left\{\begin{array}{l}
c_{o}=(1+2 \beta)\left(r+\mu^{2}\right)^{2} \\
c_{1}=2\left(r(1+r+(2+r) \beta)+(2 r(1+\beta)-1) \mu^{2}+(1+\beta) \mu^{4}\right) \\
c_{2}=1-2 \beta+r(4+r+4 \beta)+2(r+2 \beta) \mu^{2}+\mu^{4}+\frac{4 r^{2}}{\left(r+\mu^{2}\right)^{2}}+\frac{4 r(-1+\beta)}{r+\mu^{2}} \\
c_{3}=2\left(-1+r+\beta+\mu^{2}+\frac{2 r}{r+\mu^{2}}\right) \\
c_{4}=1
\end{array}\right.
$$

Now, by using Routh-Hurwitz stability criteria for four dimensional system we have the following Routh array [20]:

$$
R_{a}=\left(\begin{array}{cccc}
c_{4} & c_{2} & c_{0} & 0 \\
c_{3} & c_{1} & 0 & 0 \\
\frac{c_{2} c_{3}-c_{1} c_{4}}{c_{3}} & c_{0} & 0 & 0 \\
\frac{-c_{0} c_{3}^{2}+c_{1}\left(c_{2} c_{3}-c_{1} c_{4}\right)}{c_{2} c_{3}-c_{1} c_{4}} & 0 & 0 & 0 \\
c_{0} & 0 & 0 & 0
\end{array}\right)
$$

where $c_{i}$ 's for $i=0,1,2,3,4$ are given in (15). For stability of system (6) about $\left(\frac{\mu}{r+\mu^{2}}, \mu, \frac{\mu}{r+\mu^{2}}, \mu\right)$, it is necessary that all the elements in column one of $R_{a}$ have same sign [20]. Hence, we have the following result for the local stability of system (6) about $\left(\frac{\mu}{r+\mu^{2}}, \mu, \frac{\mu}{r+\mu^{2}}, \mu\right)$.

Theorem 3.1. [20] Assume the fourth degree characteristic equation

$$
c_{4} \xi^{4}+c_{3} \xi^{3}+c_{2} \xi^{2}+c_{1} \xi+c_{0}=0
$$

where $c_{0}, c_{1}, c_{2}, c_{3}$ and $c_{4}$ are given in (15). Additionally, let $\xi_{1}, \xi_{2}, \xi_{3}, \xi_{4}$ are roots of (16) and $D_{2}$ be any open disk of radius one. Then, the necessary and sufficient conditions that $\xi_{1}, \xi_{2}, \xi_{3}, \xi_{4} \in D_{2}$ are given as:

$$
\left\{\begin{array}{l}
c_{0}>0 \\
c_{3}>0 \\
c_{1}\left(c_{2} c_{3}-c_{1} c_{4}\right)-c_{0} c_{3}^{2}>0 \\
c_{4}>0
\end{array}\right.
$$

Theorem 3.2. Assume the fourth degree characteristic equation

$$
c_{4} \xi^{4}+c_{3} \xi^{3}+c_{2} \xi^{2}+c_{1} \xi+c_{0}=0,
$$


where

$$
\left\{\begin{array}{l}
c_{o}=(1+2 \beta)\left(r+\mu^{2}\right)^{2} \\
c_{1}=2\left(r(1+r+(2+r) \beta)+(2 r(1+\beta)-1) \mu^{2}+(1+\beta) \mu^{4}\right), \\
c_{2}=1-2 \beta+r(4+r+4 \beta)+2(r+2 \beta) \mu^{2}+\mu^{4}+\frac{4 r^{2}}{\left(r+\mu^{2}\right)^{2}}+\frac{4 r(-1+\beta)}{r+\mu^{2}}, \\
c_{3}=2\left(-1+r+\beta+\mu^{2}+\frac{2 r}{r+\mu^{2}}\right) \\
c_{4}=1 .
\end{array}\right.
$$

Additionally, let $\xi_{1}, \xi_{2}, \xi_{3}, \xi_{4}$ are roots of (18) and $D_{2}$ be any open disk of radius one. Then, $\xi_{1}, \xi_{2}, \xi_{3}, \xi_{4} \in D_{2}$ if and only if $\beta>0, r>0$ and $\mu \geq 1$.

Proof. Assume that $J^{*}\left(u_{*}, v_{*}, w_{*}, z_{*}\right)$ be the jacobian matrix of system (6) about $\left(u_{*}, v_{*}\right.$, $\left.w_{*}, z_{*}\right)$. In addition, suppose $F(\xi)=0$ be the characteristic equation obtained from $J^{*}\left(u_{*}, v_{*}, w_{*}, z_{*}\right)$. Then, $F(\xi)=0$ can be specified as follows

$$
c_{4} \xi^{4}+c_{3} \xi^{3}+c_{2} \xi^{2}+c_{1} \xi+c_{0}=0
$$

where $c_{0}, c_{1}, c_{2}, c_{3}$ and $c_{4}$ are given in (19). Moreover, from $J^{*}\left(u_{*}, v_{*}, w_{*}, z_{*}\right)$ we have

$$
\left\{\begin{array}{l}
c_{o}=(1+2 \beta)\left(r+\mu^{2}\right)^{2}, \\
c_{1}\left(c_{2} c_{3}-c_{1} c_{4}\right)-c_{0} c_{3}^{2}=-4(1+2 \beta)\left(r(1+r+\beta)+(2 r+\beta-1) \mu^{2}+\mu^{4}\right)^{2} \\
+\alpha_{1}\left(\alpha_{2}+c_{3} \alpha_{3}\right), \\
c_{3}=2\left(-1+r+\beta+\mu^{2}+\frac{2 r}{r+\mu^{2}}\right), \\
c_{4}=1,
\end{array}\right.
$$

with

$$
\left\{\begin{array}{l}
\alpha_{1}=2\left(r(1+r+(2+r) \beta)+(-1+2 r(1+\beta)) \mu^{2}+(1+\beta) \mu^{4}\right), \\
\alpha_{2}=-2\left(r(1+r+(2+r) \beta)+(-1+2 r(1+\beta)) \mu^{2}+(1+\beta) \mu^{4}\right), \\
\alpha_{3}=1-2 \beta+r(4+r+4 \beta)+2(r+2 \beta) \mu^{2}+\mu^{4}+\frac{4 r^{2}}{\left(r+\mu^{2}\right)^{2}}+\frac{4 r(-1+\beta)}{r+\mu^{2}}
\end{array}\right.
$$

Finally, if we have $\beta>0, r>0$ and $\mu \geq 1$ then we have

$$
c_{0}, c_{1}\left(c_{2} c_{3}-c_{1} c_{4}\right)-c_{0} c_{3}^{2}, c_{3}>0
$$

Consequently, all the conditions of array (15) are satisfied. Which completes the proof of theorem.

\section{Neimark-Scaker bifurcation}

Bifurcation analysis in discrete-time systems is a key attention of current studies due to the complexity in these systems. Existence of bifurcation is occasionally disapproving in 
any mathematical model, because there might be unpredictability and annihilation due to chaos [21]. In this part of article, we examine the parametric situations for the presence of Neimark-Scaker bifurcation for one and only positive fixed point $\left(u_{*}, v_{*}, w_{*}, z_{*}\right)$ of system (8). In any time dependent mathematical system, when a specific parameter crosses its critical value, numerous varieties of bifurcations arise from its equilibrium point. Several dynamical properties of a structure can be deliberated due to occurrence of NeimarkScaker bifurcation. Bifurcation frequently arises when the permanence of a fixed point deviates i.e., qualitative assets of a dynamical system amend. We deliberate NeimarkScaker bifurcation for solitary positive equilibrium point $\left(u_{*}, v_{*}, w_{*}, z_{*}\right)$ of structure (8) by means of an obvious standard for Neimark-Scaker bifurcation and taking $\eta$ as a parameter of bifurcation. Due to appearance of Neimark-Scaker bifurcation, locked invariant circles are formed. Homogeneously, individual can catch some inaccessible orbits of periodic performance along with paths that shield the invariant circle compactly. The bifurcation may be subcritical or supercritical causing in a unstable or stable locked invariant curve, correspondingly. In command to study the Neimark-Sacker bifurcation in system (8), we have the next obvious standard of Hopf bifurcation [22]. By means of this standard one can catch the presence of Neimark-Scaker bifurcation deprived of finding the eigenvalues.

Lemma 4.1. [22] Let $Z_{k+1}=g_{r}\left(Z_{k}\right)$ be a discrete dynamical system of dimension $n$, where $r \in \Re$ is bifurcation parameter. Let $Z_{*}$ be equilibrium point of mapping $g_{r}$, and characteristic equation of variational matrix $J_{\left(Z_{*}\right)}=\left[s_{i j}\right]_{n \times n}$ of $n$-dimensional function $g_{r}\left(Z_{k}\right)$ can be written as:

$$
F_{r}(\xi)=\xi^{n}+d_{1} \xi^{n-1}+d_{2} \xi^{n-2}+\ldots \ldots+d_{n-1} \xi+d_{n}
$$

where, $d_{i}=d_{i}(r, v), i=1,2,3, \ldots \ldots, n$ and $v$ is control parameter or any other parameter which is to be determined. Furthermore, suppose that $\square_{0}^{ \pm}(r, v)=1, \square_{1}^{ \pm}(r, v), \ldots \ldots, \square_{n}^{ \pm}(r, v)$ be the progression of determinants defined by $\square_{i}^{ \pm}(r, v)=\operatorname{det}\left(A_{1} \pm A_{2}\right), i=1,2,3, \ldots \ldots, n$, where

$$
A_{1}=\left(\begin{array}{ccccc}
1 & d_{1} & d_{2} & \ldots & d_{i-1} \\
0 & 1 & d_{1} & \ldots . & d_{i-2} \\
0 & 0 & 1 & \ldots . & d_{i-3} \\
\ldots & \ldots & \ldots & \ldots . & \ldots . \\
0 & 0 & 0 & \ldots . & 1
\end{array}\right)
$$


and

$$
A_{2}=\left(\begin{array}{ccccc}
d_{n-i+1} & d_{n-i+2} & \ldots & d_{n-1} & d_{n} \\
d_{n-i+2} & d_{n-i+2} & \ldots & d_{n} & 0 \\
\ldots & \ldots & \ldots & \ldots & \ldots \\
d_{n-1} & d_{n} & 0 & 0 & 0 \\
d_{n} & 0 & 0 & \ldots & 0
\end{array}\right)
$$

Furthermore, the following conditions are satisfied: $\star_{1}$ Eigenvalue assignment: $\square_{n-1}^{-}\left(r_{0}, v\right)=0, \square_{n-1}^{+}\left(r_{0}, v\right)>0, F_{r_{0}}(1)>0,(-1)^{n} F_{r_{0}}(-1)>0, \square_{i}^{ \pm}\left(r_{0}, v\right)>0, i=$ $n-3, n-5, \ldots ., 1$ or $i=n-3, n-5, \ldots . ., 2$ according to $n$ is even or odd, respectively.

Transversality condition: $\left[\frac{d\left(\square_{n-1}^{-}(r, v)\right)}{d r}\right]_{r=r_{0}} \neq 0 . \star_{3}$ Non-resonance condition: $\cos \left(\frac{2 \pi}{m}\right) \neq$ $\sigma$, or resonance $\cos \left(\frac{2 \pi}{m}\right)=\sigma$, where $m=3,4,5, \ldots$, and $\sigma=-1+\frac{0.5 F_{r_{0}}(1) \square_{n-3}^{-}\left(r_{0}, v\right)}{\square_{n-2}^{+}\left(r_{0}, v\right)}$. Then, there exist Neimark-Scaker bifurcation at $r_{0}$.

Then, by taking $\eta$ as bifurcation parameter and by using Lemma 4.1, we get the following result related to the existence of Neimark-Scaker bifurcation.

Theorem 4.1. The one and only fixed point $\left(u_{*}, v_{*}, w_{*}, z_{*}\right)$ of system (8) experiences the Neimark-Scaker bifurcation, if the next conditions are fulfilled:

$$
\left\{\begin{array}{l}
1 \pm d_{4}>0 \\
1-d_{4}-d_{2}-d_{3}{ }^{2}+d_{4}{ }^{3}-d_{4}{ }^{2}\left(1+d_{2}\right)-d_{1}{ }^{2} d_{4}+2 d_{2} d_{4}+d_{1} d_{3}\left(1+d_{4}\right)=0 \\
1+d_{4}+d_{2}-d_{3}{ }^{2}-d_{4}{ }^{3}-d_{4}{ }^{2}-d_{1}{ }^{2} d_{4}-d_{2} d_{4}^{2}-d_{1} d_{3}\left(1-d_{4}\right)>0 \\
1+d_{1}+d_{2}+d_{3}+d_{4}>0 \\
1-d_{1}+d_{2}-d_{3}+d_{4}>0
\end{array}\right.
$$

where $d_{1}, d_{2}, d_{3}, d_{4}$ are provided in (12).

Proof. By using Lemma 4.1 for a discrete-time mathematical system of 4-dimension, we have

$$
\left\{\begin{aligned}
A_{1} & =\left(\begin{array}{ccc}
1 & d_{1} & d_{2} \\
0 & 1 & d_{1} \\
0 & 0 & 1
\end{array}\right) \\
A_{2} & =\left(\begin{array}{ccc}
d_{2} & d_{3} & d_{4} \\
d_{3} & d_{4} & 0 \\
d_{4} & 0 & 0
\end{array}\right)
\end{aligned}\right.
$$

By using Lemma 4.1 for a discrete-time mathematical system of 4-dimension and taking the characteristic equation (23) of system (8) about its one and only positive fixed point $\left(\frac{\mu}{r+\mu^{2}}, \mu, \frac{\mu}{r+\mu^{2}}, \mu\right)$, then we get the following inequalities and equalities:

$$
\left\{\begin{array}{l}
\square_{1}^{ \pm}(\eta)=1 \pm d_{4}>0, \\
\square_{3}^{-}(\eta)=1-d_{4}-d_{2}-d_{3}{ }^{2}+d_{4}{ }^{3}-d_{4}{ }^{2}\left(1+d_{2}\right)-d_{1}{ }^{2} d_{4}+2 d_{2} d_{4}+d_{1} d_{3}\left(1+d_{4}\right)=0, \\
\square_{3}^{+}(\eta)=1+d_{4}+d_{2}-d_{3}{ }^{2}-d_{4}{ }^{3}-d_{4}{ }^{2}-d_{1}{ }^{2} d_{4}-d_{2} d_{4}^{2}-d_{1} d_{3}\left(1-d_{4}\right)>0, \\
F_{\eta}(1)=1+d_{1}+d_{2}+d_{3}+d_{4}>0, \\
(-1)^{4} F_{\eta}(-1)=1-d_{1}+d_{2}-d_{3}+d_{4}>0,
\end{array}\right.
$$


which confirms the existence of Neimark-Scaker bifurcation in system (8) about $\left(\frac{\mu}{r+\mu^{2}}, \mu, \frac{\mu}{r+\mu^{2}}, \mu\right)$, whenever $\eta$ is taken as bifurcation parameter.

\section{Chaos control}

In dynamical systems, it is preferred that the system be enhanced with respect to some performance standard and chaos be avoided. In modern days, monitoring disorder and chaos in discrete-time systems is a subject of boundless attention of various investigators and applied approaches can be used in numerous fields, for example physics laboratories, communications, turbulence and cardiology [23]. In discrete-time dynamical systems, control of chaos can be acquired by expending numerous approaches, namely, pole-placement method [24], hybrid control technique [25], and state response control technique (OGY method) [26]. For additional study of these chaos control approaches for discrete-time schemes, we refer a bibliophile to [27-30] and references are therein. In this part of manuscript, we simply concentrate on a generalized hybrid control approach [32], which is centered on feedback control approach and parameter perturbation. In direction to control the Neimark-sacker bifurcation in system (8), we use generalized hybrid control approach [32]. Clearly, the modified hybrid control strategy [32] is very easy to apply and efficient as compared to other control schemes. Moreover, it is applicable to almost every type of discrete-time dynamical system and it has better results than other schemes such as, larger length of controlled intervals, wider in application, and easy for computation [32]. Consider the following an n-dimensional discrete dynamical system:

$$
s_{n+1}=g\left(s_{n}, \omega\right)
$$

where $s_{n} \in R^{n}, n \in Z$ and the bifurcation parameter for system (26) is $\omega \in R$. Suppose $\omega \in \Re$ is parameter for which system (26) experiences the bifurcation. The purpose of applying the generalized technique for controlling the bifurcation is to regain the maximum range of stable region in (26) by reduction of length of unstable region. Hence, we apply the following generalized hybrid control technique by applying state feedback along with parameter

$$
s_{n+k}=\theta^{3} g^{(k)}\left(s_{n}, \omega\right)+\left(1-\theta^{3}\right) s_{n}
$$

where $k \in N$, and $0<\theta<1$ is control parameter. $g^{(k)}$ is $k^{\text {th }}$ iteration of $g($.$) . For$ $\theta=1$ one has the original system (26). Applying technique (27) on model (8) we get the 
following controlled model:

$$
\begin{aligned}
& u_{n+1}=\theta^{3} \frac{\left(u_{n}+\eta \mu\right)}{\left(1+\eta\left(v_{n}^{2}+r\right)\right)}+\left(1-\theta^{3}\right) u_{n}, \\
& v_{n+1}=\theta^{3} \frac{v_{n}+\eta\left(u_{n} v_{n}^{2}+r u_{n}+\beta z_{n}\right)}{(1+\eta(1+\beta))}+\left(1-\theta^{3}\right) v_{n}, \\
& w_{n+1}=\theta^{3} \frac{\left(w_{n}+\eta \mu\right)}{\left(1+\eta\left(z_{n}^{2}+r\right)\right)}+\left(1-\theta^{3}\right) w_{n}, \\
& z_{n+1}=\theta^{3} \frac{\left(z_{n}+\eta\left(w_{n} z_{n}^{2}+r w_{n}+\beta v_{n}\right)\right)}{(1+\eta(1+\beta))}+\left(1-\theta^{3}\right) z_{n}
\end{aligned}
$$

Furthermore, the variational matrix $J^{*}$ for $(28)$ about $\left(u_{*}, v_{*}, w_{*}, z_{*}\right)=\left(\frac{\mu}{r+\mu^{2}}, \mu, \frac{\mu}{r+\mu^{2}}, \mu\right)$ is given as:

$$
J^{*}=\left(\begin{array}{cccc}
1+\theta^{3}\left(-1+\frac{1}{1+\eta\left(r+\mu^{2}\right)}\right) & -\frac{2 \eta \theta^{3} \mu^{2}}{\left(r+\mu^{2}\right)\left(1+\eta\left(r+\mu^{2}\right)\right)} & 0 & 0 \\
\frac{\eta \theta^{3}\left(r+\mu^{2}\right)}{1+\eta+\beta \eta} & 1-\theta^{3}+\frac{\theta^{3}\left(1+\frac{2 \eta \mu^{2}}{r+\mu^{2}}\right)}{1+\eta+\beta \eta} & 0 & j_{11} \\
0 & 0 & j_{12} & -\frac{2 \eta \theta^{3} \mu^{2}}{\left(r+\mu^{2}\right)\left(1+\eta\left(r+\mu^{2}\right)\right)} \\
0 & \frac{\beta \eta \theta^{3}}{1+\eta+\beta \eta} & \frac{\eta \theta^{3}\left(r+\mu^{2}\right)}{1+\eta+\beta \eta} & 1-\theta^{3}+\frac{\theta^{3}\left(1+\frac{2 \eta \mu^{2}}{r+\mu^{2}}\right)}{1+\eta+\beta \eta}
\end{array}\right) .
$$

Where $j_{11}=\frac{\beta \eta \theta^{3}}{1+\eta+\beta \eta}$ and $j_{12}=1+\theta^{3}\left(\frac{1}{1+\eta\left(r+\mu^{2}\right)}-1\right)$. Finally, we have the following result related to the local stability analysis of controlled system $(28)$ about $\left(u_{*}, v_{*}, w_{*}, z_{*}\right)=$ $\left(\frac{\mu}{r+\mu^{2}}, \mu, \frac{\mu}{r+\mu^{2}}, \mu\right)$.

Theorem 5.1. Consider the following biquadratic polynomial equation with real coefficients

$$
\rho_{1}^{4}+d_{1}^{*} \rho_{1}^{3}+d_{2}^{*} \rho_{1}^{2}+d_{3}^{*} \rho_{1}+d_{4}^{*}=0
$$

where (30) is obtained from $J^{*}$. Then, necessary and sufficient conditions that all the roots of (30) lie inside the disk of unit radius are given as follows:

$$
\left|d_{3}^{*}+d_{1}^{*}\right|<1+d_{4}^{*}+d_{2}^{*}, \quad\left|d_{3}^{*}-d_{1}^{*}\right|<2\left(1-d_{4}^{*}\right), \quad d_{2}^{*}-3 d_{4}^{*}<3,
$$

and

$$
d_{4}^{*}+d_{2}^{*}+d_{4}^{* 2}+d_{3}^{* 2}+d_{4}^{* 2} d_{2}^{*}+d_{4}^{*} d_{1}^{* 2}<1+2 d_{4}^{*} d_{2}^{*}+d_{3}^{*} d_{1}^{*}+d_{4}^{*} d_{3}^{*} d_{1}^{*}+d_{4}^{* 3} .
$$

\section{Numerical Simulation}

In this part of article the numerical study of dynamics of (8) is provided. 
Example 6.1. Assume that $r=0.0698, \beta=0.3469, \mu=0.97993, u_{0}=0.954166, v_{0}=$ $0.97993, w_{0}=0.954166, z_{0}=0.97993$ and $\left.\left.\eta \in\right] 0,1\right]$. Then, the mathematical system (8) takes the following form:

$$
\begin{aligned}
u_{n+1} & =\frac{\left(u_{n}+\eta 0.97993\right)}{\left(1+\eta\left(v_{n}^{2}+0.0698\right)\right)}, \\
v_{n+1} & =\frac{v_{n}+\eta\left(u_{n} v_{n}^{2}+0.0698 u_{n}+0.3469 z_{n}\right)}{(1+\eta(1+0.3469))}, \\
w_{n+1} & =\frac{\left(w_{n}+\eta 0.97993\right)}{\left(1+\eta\left(z_{n}^{2}+0.0698\right)\right)}, \\
z_{n+1} & =\frac{\left(z_{n}+\eta\left(w_{n} z_{n}^{2}+0.0698 w_{n}+0.3469 v_{n}\right)\right)}{(1+\eta(1+0.3469))} .
\end{aligned}
$$

Additionally, in this case the one and only positive fixed point is $(0.954166,0.97993,0.954166,0.97993)$. For aforementioned values of parameters one can obtain the jacobian matrix $J(0.954,0.979,0.954,0.979)$ as follows:

$$
J(0.954166,0.97993,0.954166,0.97993)=\left(\begin{array}{cccc}
0.7576 & -0.43873 & 0 & 0 \\
0.22557 & 1.1133 & 0 & 0.07596 \\
0 & 0 & 0.75761 & -0.438739 \\
0 & 0.07596 & 0.22557 & 1.11334
\end{array}\right) .
$$

The characteristic polynomial $P(\rho)$ calculated from $J(0.95416,0.9799,0.95416,0.9799)$ is given by

$$
P(\rho)=0.884894-3.5178 \rho+5.37959 \rho^{2}-3.74191 \rho^{3}+\rho^{4} .
$$

Moreover, roots of $P(\rho)=0$ are given as

$$
\begin{gathered}
\rho_{1}=0.897493-0.28178 \iota, \rho_{2}=0.897493+0.28178 \iota, \rho_{3}=0.97346-0.228858 \iota, \\
\rho_{4}=0.97346+0.228858 \iota,
\end{gathered}
$$

with $\left|\rho_{3,4}\right|=1,\left|\rho_{1,2}\right| \neq 1, d_{1}=-3.7419, d_{2}=5.37958, d_{3}=-3.5178$, and $d_{4}=0.884893$.

$x$

$$
\left\{\begin{array}{l}
\square_{1}^{+}(\eta)=1+d_{4}=1.88489>0, \\
\square_{1}^{-}(\eta)=1-d_{4}=0.115107>0, \\
\square_{3}^{-}(\eta)=1-d_{4}-d_{2}-d_{3}^{2}+d_{4}{ }^{3}-d_{4}^{2}\left(1+d_{2}\right)-d_{1}^{2} d_{4}+2 d_{2} d_{4}+d_{1} d_{3}\left(1+d_{4}\right)=0, \\
\square_{3}^{+}(\eta)=1+d_{4}+d_{2}-d_{3}{ }^{2}-d_{4}{ }^{3}-d_{4}^{2}-d_{1}^{2} d_{4}-d_{2} d_{4}^{2}-d_{1} d_{3}\left(1-d_{4}\right)=0.0761663>0, \\
F_{\eta}(1)=1+d_{1}+d_{2}+d_{3}+d_{4}=0.00477238>0, \\
(-1)^{4} F_{\eta}(-1)=1-d_{1}+d_{2}-d_{3}+d_{4}=4.52418>0 .
\end{array}\right.
$$




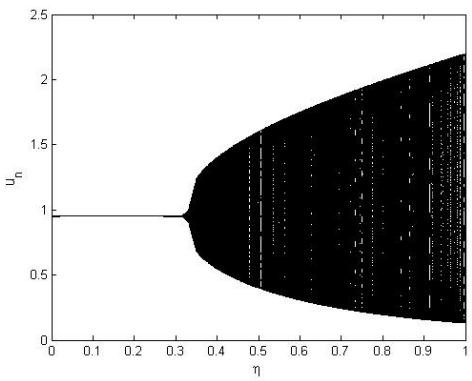

(a)

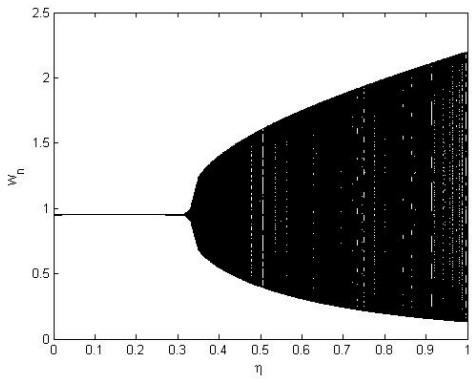

(c)

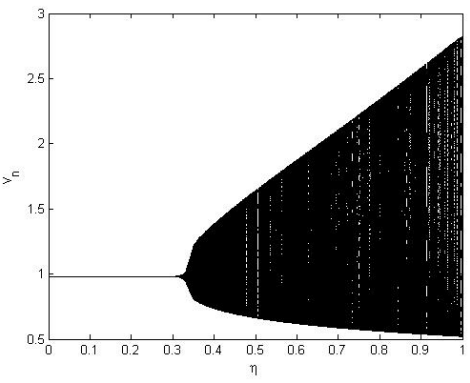

(b)

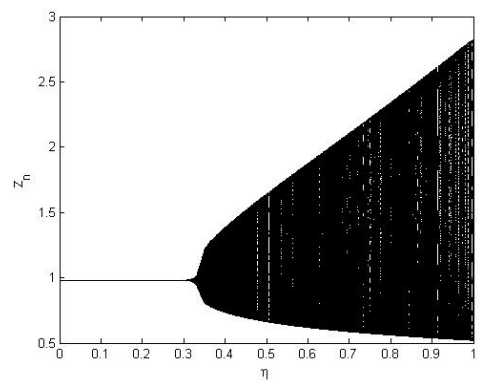

(d)

Figure 1. Bifurcation diagrams for system (8) for $r=0.0698, \beta=0.3469, \mu=$ $0.97993, u_{0}=0.954166, v_{0}=0.97993, w_{0}=0.954166, z_{0}=0.97993$ and $\eta \in] 0,1]$

Hence, all the conditions for existence of Neimark-sacker bifurcation is satisfied (see Theorem 4.1). In this case the graphical behavior of each concentration variable is shown in Fig.1. In Fig.2 some phase portraits are given for variation of $\eta$ in ]0,1[. Hence, it can be easily seen that there exists the Neimark-sacker bifurcation when $\eta$ certainly passes through $\eta=0.310602$ (see Fig. 2).

Example 6.2. Assume that $\eta \in] 0,1], r=0.0698, \beta=0.3469, \mu=0.97993, u_{0}=$ $0.954166, v_{0}=0.97993, w_{0}=0.954166, z_{0}=0.97993$ and $\left.\left.\theta \in\right] 0,1\right]$. Then, the mathe- 
matical system (28) takes the following form:

$$
\begin{aligned}
& u_{n+1}=\theta^{3} \frac{\left(u_{n}+\eta 0.97993\right)}{\left(1+\eta\left(v_{n}^{2}+0.0698\right)\right)}+\left(1-\theta^{3}\right) u_{n}, \\
& v_{n+1}=\theta^{3} \frac{v_{n}+\eta\left(u_{n} v_{n}^{2}+0.0698 u_{n}+0.3469 z_{n}\right)}{(1+\eta(1+0.3469))}+\left(1-\theta^{3}\right) v_{n}, \\
& w_{n+1}=\theta^{3} \frac{\left(w_{n}+\eta 0.97993\right)}{\left(1+\eta\left(z_{n}^{2}+0.0698\right)\right)}+\left(1-\theta^{3}\right) w_{n}, \\
& z_{n+1}=\theta^{3} \frac{\left(z_{n}+\eta\left(w_{n} z_{n}^{2}+0.0698 w_{n}+0.3469 v_{n}\right)\right)}{(1+\eta(1+0.3469))}+\left(1-\theta^{3}\right) z_{n} .
\end{aligned}
$$

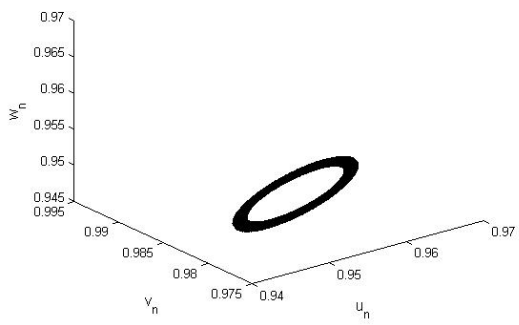

(a)

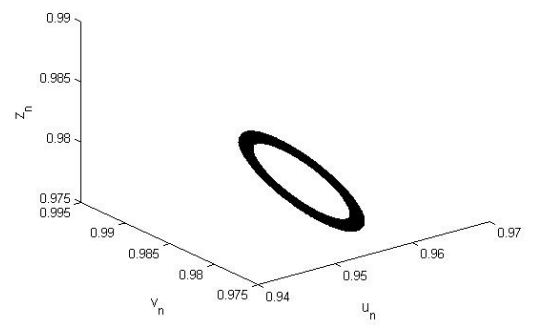

(b)

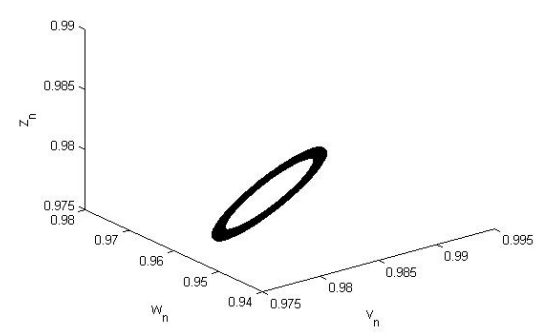

(c)

Figure 2. Phase portraits for system (8) for $r=0.0698, \beta=0.3469, \mu=0.97993$, $u_{0}=0.954166, v_{0}=0.97993, w_{0}=0.954166, z_{0}=0.97993$ and $\left.\left.\eta \in\right] 0,1\right]$

Additionally, in this case the one and only positive fixed point is

$(0.954166,0.97993,0.954166,0.97993)$. For aforementioned values of parameters one can obtain the jacobian matrix $J(0.954,0.979,0.954,0.979)$ as follows:

$$
J(0.95416,0.97993,0.95416,0.97993)=\left(\begin{array}{cccc}
0.691371 & -0.558636 & 0 & 0 \\
0.270997 & 1.13617 & 0 & 0.0912651 \\
0 & 0 & 0.691371 & -0.558636 \\
0 & 0.0912651 & 0.270997 & 1.13617
\end{array}\right) .
$$


The characteristic polynomial $P(\rho)$ calculated from $J(0.954166,0.97993,0.954166$, $0.97993)$ is given by

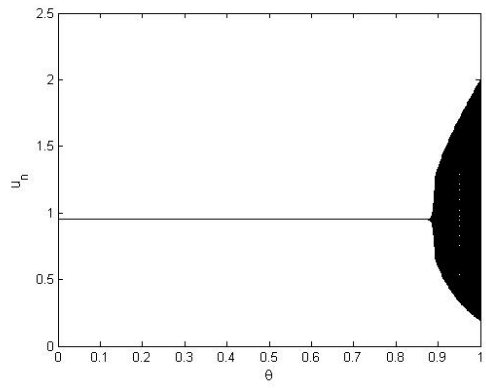

(a)

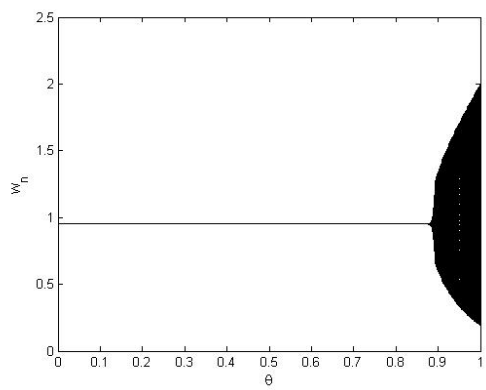

(c)

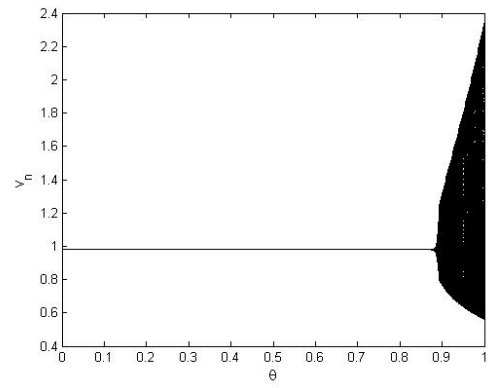

(b)

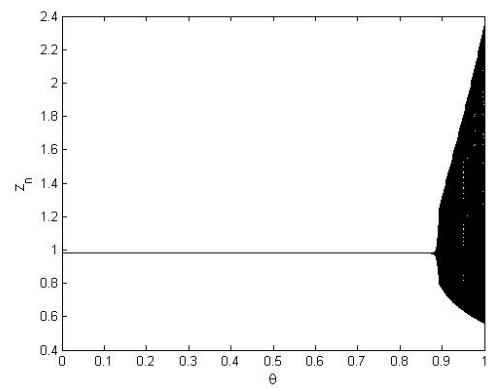

(d)

Figure 3. Controlled diagrams for system (33) for $\eta \in] 0,1], r=0.0698, \beta=$ $0.3469, \mu=0.97993, u_{0}=0.954166, v_{0}=0.97993, w_{0}=0.954166, z_{0}=$ 0.97993 and $\theta \in] 0,1]$

$$
P(\rho)=0.873804-3.41293 \rho+5.20537 \rho^{2}-3.65508 \rho^{3}+\rho^{4} .
$$

Moreover, in this case the equilibrium point $(0.954166,0.97993,0.954166,0.97993)$ of system (33) is stable for

$$
0<\theta<0.8809233539967847 \text {. }
$$

In this case the graphical behavior of each concentration variable is shown in Fig.3. Hence, it can be easily seen that bifurcation is controlled for maximum range of controlled parameter $\theta$ for $\eta \in] 0,1]$ (see Fig.3). Moreover, in Fig.4 some phase portraits are given for variation of $\theta$ in $] 0,1[$. Hence, it can be easily seen that there exists the Neimark-sacker 
bifurcation when $\theta$ certainly passes through $\theta=0.8809233539967847$ (see Fig. 4). In addition, there is no chance of Neimark-sacker bifurcation for $0<\theta<0.8809233539967847$ (see Fig. 4(a-c)), and for $0.8809233539967847 \leq \theta<1$ the existence of Neimark-sacker bifurcation in system (33) can be seen easily (see Fig. 4(d-f)).

Example 6.3. Assume that $r=0.0698, \beta=0.3469, \mu=0.9999, u_{0}=1.0007, v_{0}=$ $0.97993, w_{0}=1.0007, z_{0}=0.97993$. Then, the mathematical system (6) takes the following form:

$$
\begin{aligned}
& \frac{d u}{d t}=0.97993-u v^{2}-0.0698 u, \\
& \frac{d v}{d t}=u v^{2}+0.0698 u-v+0.3469(z-v), \\
& \frac{d w}{d t}=0.97993-w z^{2}-0.0698 w, \\
& \frac{d z}{d t}=w z^{2}+0.0698 w-z+0.3469(v-z),
\end{aligned}
$$

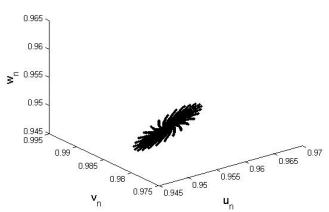

(a)

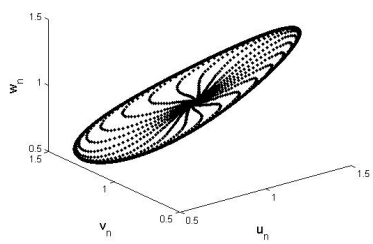

(d)

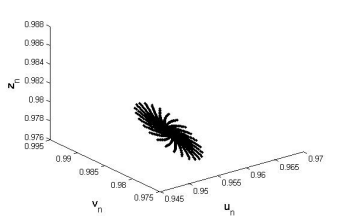

(b)

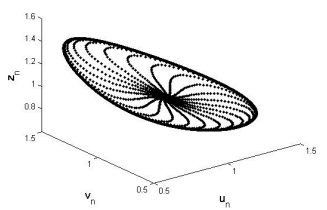

(e)

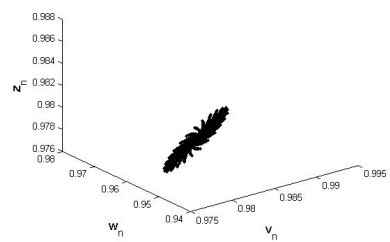

(c)

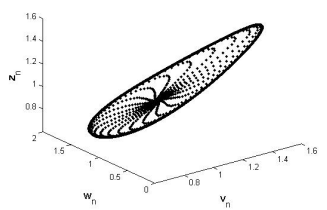

(f)

Figure 4. Phase portraits for system (33) for $\eta \in] 0,1], r=0.0698, \beta=0.3469, \mu=$ $0.97993, u_{0}=0.954166, v_{0}=0.97993, w_{0}=0.954166, z_{0}=0.97993$ and $\theta \in] 0,1]$ 


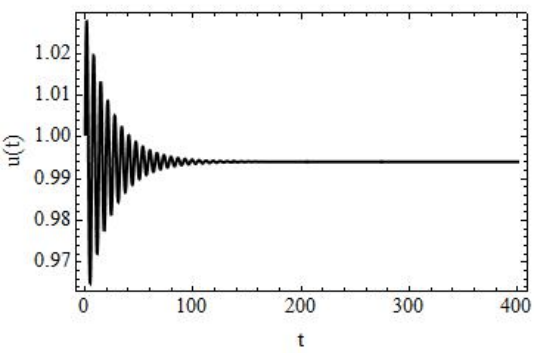

(a)

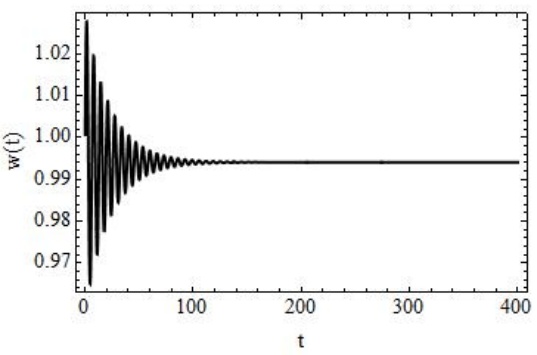

(c)

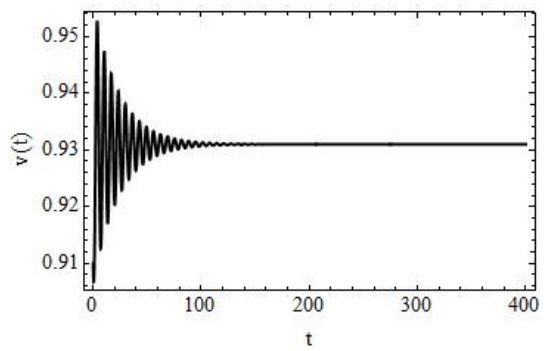

(b)

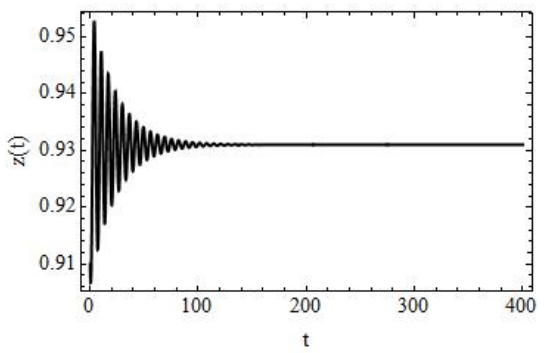

(d)

Figure 5. Plots of system (35) for $r=0.0698, \beta=0.3469, \mu=0.9999, u_{0}=$ $1.0007, v_{0}=0.97993, w_{0}=1.0007, z_{0}=0.97993$

Additionally, in this case the one and only positive fixed point is

$(1.0007,0.9793,1.0007,0.9793)$. For aforementioned values of parameters one can obtain the jacobian matrix $J(1.0007,0.9793,1.0007,0.9793)$ as follows:

$$
J(1.0007,0.9793,1.0007,0.9793)=\left(\begin{array}{cccc}
0.505244 & -0.990902 & 0 & 0 \\
0.417249 & 1.26176 & 0 & 0.147812 \\
0 & 0 & 0.505244 & -0.990902 \\
0 & 0.147812 & 0.417249 & 1.26176
\end{array}\right) .
$$

The characteristic polynomial $P(\rho)$ calculated from $J(1.0007,0.9793,1.0007,0.9793)$ is given by

$$
P(\rho)=1.09892-3.692 \rho+5.20237 \rho^{2}-3.53402 \rho^{3}+\rho^{4} .
$$

In this case the graphical behavior of each concentration variable is shown in Fig.5. Moreover, in Fig.6 some phase portraits are given for variation of $r$. Hence, it can be easily seen that there exists the system (35) remains stable about one and only fixed point 
$(1.0007,0.9793,1.0007,0.9793)$. In addition, a three dimensional phase portrait is given in Fig. 6a.

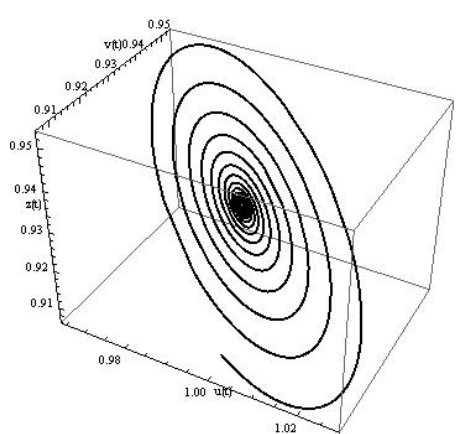

(a)

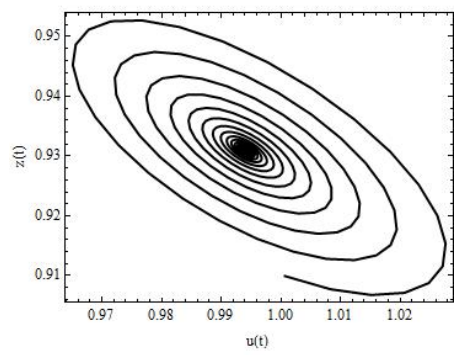

(c)

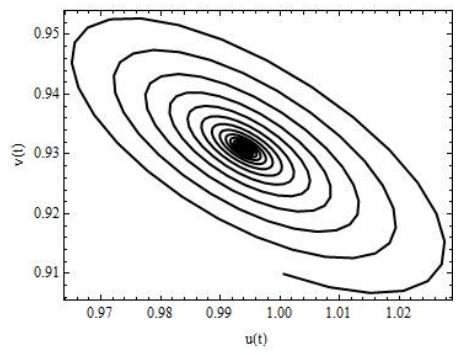

(b)

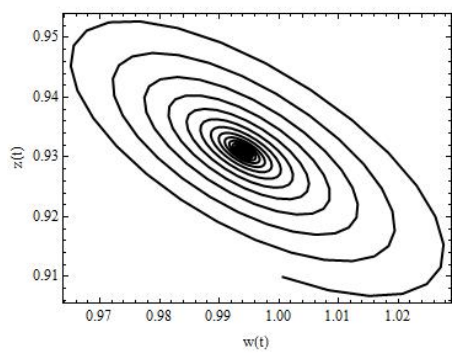

(d)

Figure 6. Phase portraits for system (35) for $r=0.0698, \beta=0.3469, \mu=0.9999$, $u_{0}=1.0007, v_{0}=0.97993, w_{0}=1.0007, z_{0}=0.97993$

Example 6.4. Assume that $r=0.01275, \beta=0.3469, \mu=0.95993, u_{0}=1.0007, v_{0}=$ 0.97993, $w_{0}=1.0007, z_{0}=0.97993$. Then, the mathematical system (6) takes the following form:

$$
\begin{aligned}
& \frac{d u}{d t}=0.97993-u v^{2}-0.01275 u \\
& \frac{d v}{d t}=u v^{2}+0.01275 u-v+0.3469(z-v), \\
& \frac{d w}{d t}=0.97993-w z^{2}-0.01275 w \\
& \frac{d z}{d t}=w z^{2}+0.01275 w-z+0.3469(v-z) .
\end{aligned}
$$

Additionally, in this case the one and only positive fixed point is $(1.0007,0.9793,1.0007,0.9793)$. For aforementioned values of parameters one can obtain the jacobian matrix 


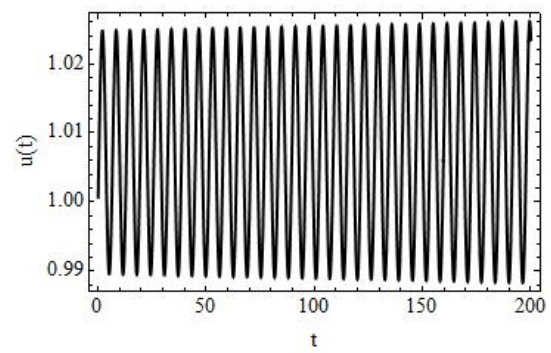

(a)

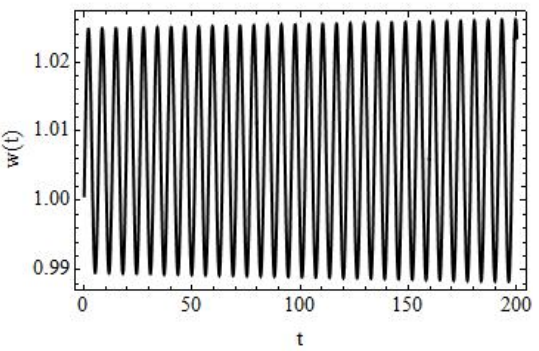

(c)

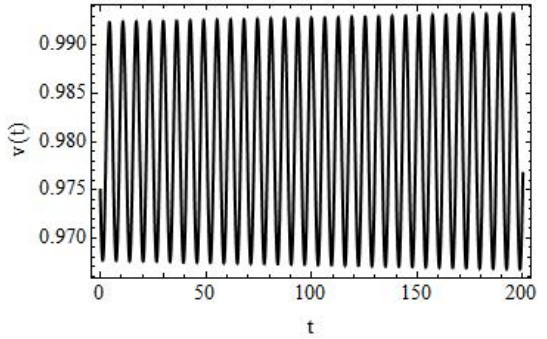

(b)

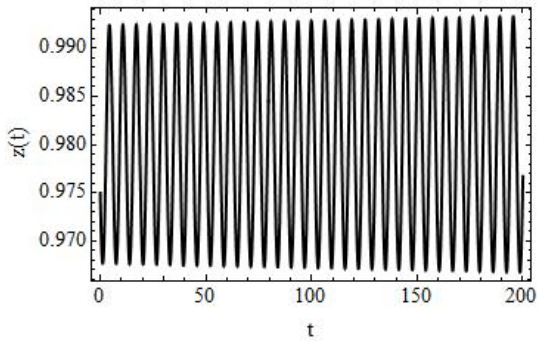

(d)

Figure 7. Plots of system (5) for $r=0.01275, \beta=0.3469, \mu=0.95993, u_{0}=$ $1.0007, v_{0}=0.97993, w_{0}=1.0007, z_{0}=0.97993$

$J(1.0007,0.9793,1.0007,0.9793)$ as follows:

$$
J(1.0007,0.9793,1.0007,0.9793)=\left(\begin{array}{cccc}
0.506839 & -1.0004 & 0 & 0 \\
0.414595 & 1.26712 & 0 & 0.147812 \\
0 & 0 & 0.506839 & -1.0004 \\
0 & 0.147812 & 0.414595 & 1.26712
\end{array}\right) .
$$

The characteristic polynomial $P(\rho)$ calculated from $J(1.0007,0.9793,1.0007,0.9793)$ is given by

$$
P(\rho)=1.1116-3.72793 \lambda+5.23903 \lambda^{2}-3.54791 \lambda^{3}+\lambda^{4} .
$$

In this case the graphical behavior of each concentration variable is shown in Fig. \%. Moreover, in Fig.8 some phase portraits are given for variation of $r$. Hence, it can be easily seen that there exists the Hopf bifurcation for system (5) about one and only fixed point $(1.0007,0.9793,1.0007,0.9793)$ when the parameter $r$ certainly passes through 0.01275 . In addition, a three dimensional phase portrait is given in Fig. 8 a. 


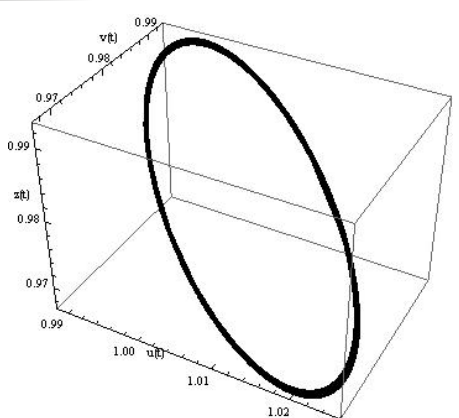

(a)

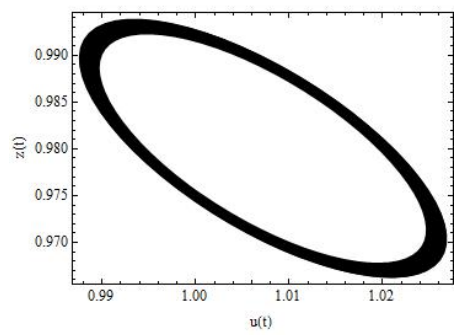

(c)

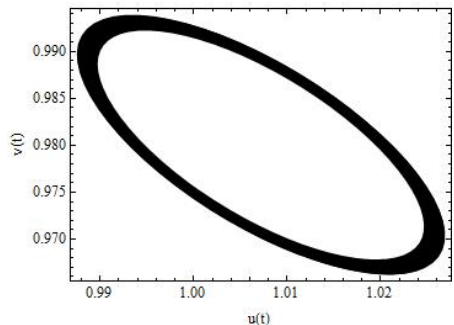

(b)

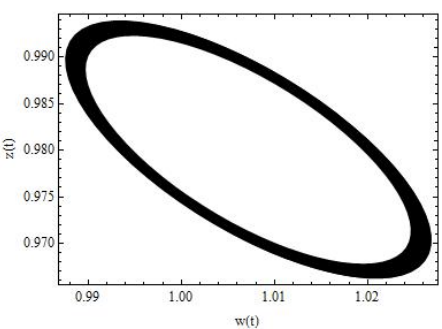

(d)

Figure 8. Phase portraits for system (5) for $r=0.01275, \beta=0.3469, \mu=0.95993$ , $u_{0}=1.0007, v_{0}=0.97993, w_{0}=1.0007, z_{0}=0.97993$

\section{Conclusion}

In this manuscript we have studied a two-cell joined cubic autocatalator chemical reaction model [1]. Additionally, during the making of basic structure of model, feedback in every cell is considered to be centered on the cubic autocatalator under the effects of pooled-chemical estimations. We have considered the pairing among the cells through autocatalyst $B$. We have discussed the local stability of continuous system (6) by using Routh-Hurwitz stability criteria. Moreover, we discretize the model (6) by using Euler's forward method and then by using a nonstandard difference scheme we obtained a consistent discrete-time counterpart of four-dimensional cubic autocatalator chemical reaction model (7). It is proved that when the pairing among the cells is taken through autocatalyst $B$ then the system (8) has only one fixed point. We have discussed the parametric conditions aimed at the local asymptotic stability of one and only positive fixed point of system (8). It is proved that the system (8) experiences the Neimark-Sacker 
bifurcation for bifurcation parameter $\eta$ at one and only positive fixed point by using an obvious standard for Neimark-Sacker bifurcation. The discrete-time counterpart (8) of original four-dimensional system (6) have shown chaotic dynamics at different standards of bifurcation parameter $\eta$. Furthermore, we have controlled the Neimark-Sacker bifurcation and chaos by using a generalized hybrid control scheme, which is centered on parameter perturbation and feedback control by taking $\theta$ as control parameter. Finally, we provided some numerical examples to demonstrate theoretical consequences. From, numerical study of (6) and (8) one can see that the system (6) remains stable for $\beta, r>0$ and $\mu \geq 1$ whereas the system (8) remains unstable under these parametric conditions. Moreover, through numerical study of system (8) there is no chance for the existence of period-doubling bifurcation, which verifies the consistency of nonstandard difference scheme [18] for mathematical system (6).

\section{References}

[1] J. A. Leach, J. H. Merkin, S. K. Scott, Two-cell coupled cubic autocatalator: the effect of the uncatalysed reaction, Dyn. Stability Syst. 7 (1992) 245-266.

[2] L. K. Forbes, B. F. Gray, Forced oscillations in an exothermic chemical reaction, Dyn. Stability Syst. 9 (1994) 253-269.

[3] B. F. Gray, M. J. Roberts, J. H. Merkin, The cubic autocatalator: The influence of the quadratic autocatalytic and the uncatalysed reactions, J. Engin. Math. 22 (1988) 267-284.

[4] B. F. Gray, J. H. Merkin, M. J. Roberts, On the structural stability of the cubic autocatalytic scheme in a closed vessel, Dyn. Stability Syst. 4 (1989) 31-54.

[5] P. Gray, S. R. Kay, S. K. Scott, Oscillations of an exothermic reaction in a closed system-I. Approximate (exponential) representation of Arrhenius temperaturedependence, Proc. Math. Phys. Eng. Sci. 416 (1988) 321-341.

[6] J. H. Merkin, D. J. Needham, S. K. Scott, On the creation, growth and extinction of oscillatory solutions for a simple pooled chemical reaction scheme, SIAM J. Appl. Math. 47 (1987) 1040-1060.

[7] J. H. Merkin, D. J. Needham, S. K. Scott, On the structural stability of a simple pooled chemical system, J. Engin. Math. 21 (1987) 115-127.

[8] J. J. Tyson, Some further studies of non-linear oscillations in chemical systems, J. Chem. Phys. 58 (1973) 3919-3930. 
[9] M. Golubitsky, W. F. Langford, Classification and unfoldings of degenerate Hopf bifurcations, J. Diff. Eq. 41 (1981) 373-415.

[10] J. J. Tyson, S. Kauffman, Control of mitosis by continuous biochemical oscillations: synchronization. Spatially inhomogeneous oscillations, J. Math. Biol. 1 (1975) 289310.

[11] J. H. Merkin, D. J. Needham, S. K. Scott, Oscillatory chemical reactions in closed vessels, Proc. R. Soc. Lond., A Math. Phys. Sci. 406 (1986) 299-323.

[12] J. A. Leach, J. H. Merkin, S. K. Scott, An analysis of a two-cell coupled nonlinear chemical oscillator, Dyn. Stability Syst. 6 (1991) 341-366.

[13] M. Ashkenazi, H. G. Othmer, Spatial patterns in coupled biochemical oscillators, J. Math. Biol. 5 (1977) 305-350.

[14] Q. Din, Bifurcation analysis and chaos control in discrete-time glycolysis models, $J$. Math. Chem. 56 (2018) 904-931.

[15] Q. Din, T. Donchev, D. Kolev, Stability, bifurcation analysis and chaos control in chlorine dioxide-iodine-malonic acid reaction, MATCH Commun. Math. Comput. Chem. 79 (2018) 577-606.

[16] X. Liu, A note on the existence of periodic solutions in discrete predator-prey models, Appl. Math. Model. 34 (2010) 2477-2483.

[17] H. I. Freedman, Deterministic Mathematical Models in Population Ecology, Marcel Dekker Incorporated, 1980.

[18] R. Mickens, Nonstandard Finite Difference Methods of Differential Equations, World Scientific, Singapore, 1994.

[19] E. A. Grove, G. Ladas, Periodicities in Nonlinear Difference Equations, CRC Press, Boca Raton, 2004.

[20] E. J. Routh, A Treatise on the Stability of a Given State of Motion, Particularly Steady Motion: Being the Essay to which the Adams Prize was Adjudged in 1877, in the University of Cambridge, Macmillan and Company, 1877.

[21] M. S. Khan, M. Samreen, M. Ozair, T. Hussain and J. F. Gomez-Aguilar, Bifurcation analysis of a discrete-time compartmental model for hypertensive or diabetic patients exposed to COVID-19, Eur. Phys, J. Plus. 156 (2021).

[22] G. Wen, Criterion to identify Hopf bifurcations in maps of arbitrary dimension, Phys. Rev. E 72 (2005) \#026201. 
[23] S. Lynch, Dynamical Systems with Applications Using Mathematica, Birkhäuser, Boston, 2007.

[24] F. J. Romeiras, C. Grebogi, E. Ott, W. P. Dayawansa, Controlling chaotic dynamical systems, Phys. D: Nonlin. Phenom. 58 (1992) 165-192.

[25] X. S. Luo, G. Chen, B. H. Wang, J. Q. Fang, Hybrid control of period-doubling bifurcation and chaos in discrete nonlinear dynamical systems, Chaos Soliton. Fract. 18 (2003) 775-783.

[26] E. Ott, C. Grebogi, J. A. Yorke, Erratum: Controlling chaos, Phys. Rev. Lett. 64 (1990) 2837.

[27] S. Parthasarathy, Homoclinic bifurcation sets of the parametrically driven Duffing oscillator, Phys. Rev. A 46 (1992) 21-47.

[28] L. Molgedey, J. Schuchhardt, H. G. Schuster, Suppressing chaos in neural networks by noise, Phys. Rev. Lett. 69 (1992) 17-37.

[29] M. S. Shabbir, Q. Din, M. Safeer, M. A. Khan, K. Ahmad, A dynamically consistent nonstandard finite difference scheme for a predator-prey model, Adv. Diff. Eq. (2019) $1-17$.

[30] A. D. Silva, P. C. Rech, Chaos and periodicity in a discrete-time Baier-Sahle model, Asian J. Math. Comput. Res. Arch. 15 (2017) 123-130.

[31] C. C. Felicio, P. C. Rech, Arnold tongues and the devil's staircase in a discrete-time Hindmarsh-Rose neuron model, Phys. Rev. A 379 (2015) 2845-2847.

[32] M. S. Khan, Stability, bifurcation and chaos control in a discrete-time prey-predator model with Holling type-II response, Netw. Biol. 9 (2019) \#58. 